\title{
ANALYSIS OF STOCHASTIC PROCESS TO MODEL SAFETY RISK IN CONSTRUCTION INDUSTRY
}

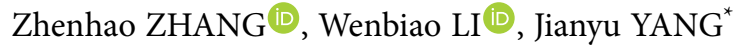 \\ School of Civil Engineering, Changsha University of Science and Technology, Changsha, China
}

Received 3 March 2020; accepted 27 October 2020

\begin{abstract}
There are many factors leading to construction safety accident. The rule presented under the influence of these factors should be a statistical random rule. To reveal those random rules and study the probability prediction method of construction safety accident, according to stochastic process theory, general stochastic process, Markov process and normal process are respectively used to simulate the risk-accident process in this paper. First, in the general-random-process-based analysis the probability of accidents in a period of time is calculated. Then, the Markov property of the construction safety risk evolution process is illustrated, and the analytical expression of probability density function of first-passage time of Markov-based risk-accident process is derived to calculate the construction safety probability. In the normal-process-based analysis, the construction safety probability formulas in cases of stationary normal risk process and non-stationary normal risk process with zero mean value are derived respectively. Finally, the number of accidents that may occur on construction site in a period is studied macroscopically based on Poisson process, and the probability distribution of time interval between adjacent accidents and the time of the $n$th accident are calculated respectively. The results provide useful reference for the prediction and management of construction accidents.
\end{abstract}

Keywords: civil engineering construction, safety accidents, probability prediction, Markov process, Normal process, Poisson process.

\section{Introduction}

Civil engineering construction has the characteristics of open air, high altitude and cross operation. Safety accidents occur from time to time because of the influence of site conditions, natural environment, temporary facilities, external environment and other factors, which has become a serious problem (Faber, 2003; Tang et al., 2012; de Lamos et al., 2004; Stewart, 2001). How to reduce the safety accidents in the process of civil engineering construction is more and more widely concerned by people, thus, the research of safety risk prediction method in civil engineering construction has important practical significance.

In recent years, scholars have conducted extensive research on the safety accidents in civil engineering construction. Golizadeh et al. (2018) created a comprehensive list of accident causes from the Loughborough Construction Accident Causation model to determine the causes of building construction accidents and related these causes to the digital engineering which has the potential to address the root causes of accidents. Irumba (2014) investigated the causes of construction accidents in Kampala, Uganda using ordinary least squares regression and spatial regression modeling, and found that the most prevalent causes of accidents are mechanical hazards, being hit by falling objects and falls from height. Zhang et al. (2019a) analyzed a typical construction accident based on four contemporary accident causation models: STAMP, AcciMap, HFACS, and the 2-4 Model, and found that the 2-4 Model could provide detailed causes of the accident and reveal a dynamic analysis and developing process. Kang and Ryu (2019) built random forest model to predict construction accident, and extracted important factors that affect the construction accident types at construction sites using feature importance. Choe and Leite (2020) proposed a safety risk generation and control model which addresses how the inherent safety risk of a worker can be transformed by different measurable risk factors. Zhang et al. (2020a) established a construction accident causation system model using accident causation theory and the system thinking method to determine critical causes of construction

*Corresponding author. E-mail: 617654991@qq.com 
accidents in China and proposed recommendations for construction safety management and accident prevention in practice. Chen et al. (2010) investigated the characteristic factors responsible for construction accident occurrence for small construction enterprises in Taiwan using methodologies of descriptive statistics, correlation coefficient analysis and ANOVA. Winge and Albrechtsen (2018), Winge et al. (2019) analyzed 176 relatively severe construction accidents using the Construction Accident Causation framework and found that risk management, immediate supervision and worker actions were key causal factors and the distribution of accident types varied regarding severity and different construction types. Chi and Han (2013) combined the systems theory with Heinrich's domino theory to explore the interrelationships of risks, and broke the chain of accident causation to analyze relationships between accidents and risks, then further identified key risk factors and risk combinations causing accidents. Besides the studies of the primary causes leading to construction accident, how to recognize safety and health hazards in advance of accidents in a construction site was also studied furtherly. Isaac and Edrei (2016) proposed a statistical model that can support a more dynamic form of safety control, the model can provide proactive alerts before potential accidents exposure and addresses risks that are the result of concurrent activities on the construction site. Lee et al. (2020) developed an audio-based event detection system which can automatically provide construction workers with prenotifications regarding safety hazards at a work zone and significantly improve the detection accuracy. Andolfo and Sadeghpour (2015) created an accident warning system that consists of an Ultra Wideband Real-Time Locating System and probabilistic accident prediction model. The probabilistic accident prediction model predicts the future construction accident by analyzing the information provided from Ultra Wideband Real-Time Locating System. Yang et al. (2012) proposed an identification system design for proactive accident prevention based on ZigBee enabled wireless sensor network (WSN), radio frequency identification (RFID) technology and an integrated ZigBee RFID sensor network structure, which greatly improved the safety of construction sites. Ayhan and Tokdmir (2019) categorized the real data about construction incidents using the Delphi method and predicted the outcome of construction incidents using conventional and artificial intelligence techniques, and proposed an effective mechanism to prevent incidents utilizing data through incident collection systems. Zhu et al. (2016) proposed novel Kalman filters for predicting the movements of the workers and mobile equipment on the construction sites so as to avoid the workers being struck by mobile equipment on sites and the effectiveness of the filters has been tested with real site videos. Based on the EUROSTAT accident model, Hoła and Szóstak (2014) proposed a comprehensive general model of the development of an accident situation that can assess the probability of various scenarios of accident events and determine the necessary preventive actions. Some research works were also done on using management methods and strategies to prevent construction accidents (Nnaji \& Karakhan, 2020; Mwakali, 2006; Shao et al., 2019; Shohet et al., 2018).

In the aspect of risk analysis and assessment, some foundational work has been done on this important topic, specifically risk-based asset integrity assessment and management. Hassan and Khan (2012) proposed a hierarchical framework used to characterize the asset and related it to an organization's strategic goal for asset integrity monitoring and assessment. Khakzad et al. (2014) proposed a methodology based on hierarchical Bayesian analysis and accident precursor data to analyze the risk of major accidents. Khakzad et al. (2012) used bow-tie (BT) approach to perform the analysis of the risk in a dynamic environment, in which the occurrence probability of accident consequences changes. Bhatia et al. (2019) presented a method to assess risk for a dynamically changing system based on continuously monitored system parameters, the calculated dynamic risk can be used to plan optimal inspection and maintenance intervals more efficiently. Ding et al. (2020) proposed a framework combining uncertainty reasoning approach and deterministic modeling approach to assess the fire accident probability before a primary accident occurs and to model the domino evolution process after a primary accident occurs, respectively. Jamot and Park (2019) compared two risk analysis methods in construction sites: System-Theoretical Process Analysis and Probabilistic Risk Analysis, and found that SystemTheoretical Process Analysis can simulate more construction scenarios and predict construction accidents more accurately.

The above research studied the causes of construction accidents, the development process of accidents, the methods to predict accidents and reduce them, etc. According to the characteristics of randomness, latency, independence and inheritance of construction accidents (Zhou et al., 2020; Li et al., 2010; Sanni-Anibire et al., 2020), this work considers the development and evolution of safety risk in the future as a random process and then study the process from risk to accident from the perspective of random process. Although great progress has been made in probabilistic risk assessment theory (Ang \& Tang, 2007; Li et al., 1993; Zhou \& Ding, 2017; Williamson \& Winget, 2005) and they have been widely used in the prediction and assessment of construction accidents, the current used probability theory is based on stochastic variable (Kang \& Ryu, 2019; Choe \& Leite, 2020; Zhang et al., 2020b; Isaac \& Edrei, 2016; Andolfo \& Sadeghpour, 2015; Jamot \& Park, 2019; Nnaji \& Karakhan, 2020), while using stochastic process theory to analyze construction safety risk problem is very rare. But actually, stochastic processes theory was widely used in many applications, such as asset integrity, fault detection and diagnosis. Don and Khan (2019) introduced a methodology for dynamic process fault detection and diagnosis based on a combined approach of hidden Markov and Bayesian network model. Arunthavanathan et al. (2020) proposed a framework combining unsupervised learning with cognitive model- 
ling to detect and diagnose unknown fault conditions. Yu et al. (2015) raised a Nonlinear Gaussian Belief Network (NLGBN) based fault diagnosis technique for industrial processes, and constructed and trained a three-layer NLGBN to extract useful features from noisy process data. Amin et al. (2019) proposed a dynamic Bayesian network (DBN) based fault detection, root cause diagnosis, and fault propagation pathway identification scheme. Amin et al. (2018) presented a hybrid methodology to detect and diagnose the faults in dynamic processes based on principal component analysis with T2 statistics and a Bayesian network. Therefore, it can be thought the studies combining stochastic process and construction risk process are meaningful and reasonable.

Various security incidents basically can be classified into two sources, unsafe state of objects and unsafe behavior of persons on construction site. For the safety accident caused by unsafe state of things, such as collapse of falsework, the stress state of key component changes during the construction process and once the stress exceeds a safety threshold the collapse accident happens. Therefore, it is reasonable to regarding the state of object such as the stress of key component of falsework as a stochastic process. Using stochastic process theory to reveal the stochastic law of how risk developing into accident is important. According to the types and characteristics of safety accidents, stochastic process models with special properties are established to simulate the development and evolution of safety risk until accident happens. This work can help to deepen our understanding of the process from risk to accident and establish theoretical basis for the prediction and control of construction accidents.

\section{Construction safety accident process simulation based on general random process}

\subsection{The analysis of construction safety probability under construction risk-accident random process}

Any future construction risk-accident process could be regarded as a random process $X(t)$ (Figure 1) because of its obvious randomness (Jin et al., 2020; Rey et al., 2011). If $X(t)$ exceeds a certain safety limit in a period of time an accident happens. The probability that $X(t)$ never exceeds the safety threshold in a given period of time $(0, T]$ is just the construction safety probability. The parameters in the stochastic process model describing the future risk-

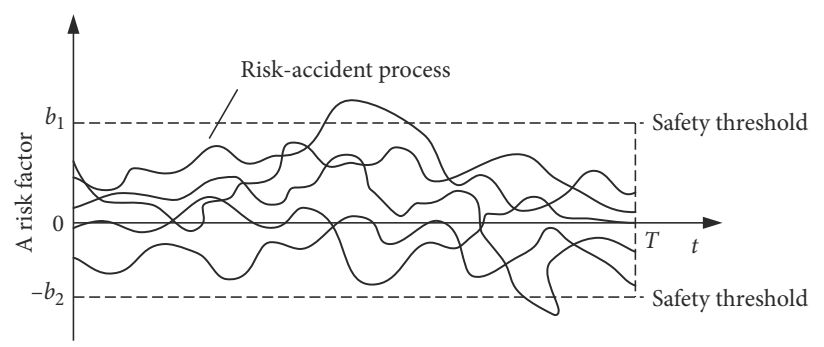

Figure 1. Future risk development process could be regarded as a random process accident process should be determined by the statistics of past accidents.

For the characteristics of construction accident phenomenon, it is easily observed that the occurrence of an accident is rare event, and the occurrences of any two accidents are independent of each other because there is no mutual influence between arbitrary two accidents. Thus, the event of the occurrence of an accident would satisfy the characteristics of a Poisson process, which can be seem as below (denote $N(t)$ as the number of construction accidents in the period of $[0, t)$ ).

1. At initial time no construction accident occurs, i.e., $N(0)=0$;

2 . For any $s \geq t \geq 0, \Delta t>0$, the number of accidents in a period of $[t+\Delta t, s+\Delta s)$ only depends on the time interval $(s+\Delta s)-(t+\Delta t)=s-t$, and are not associated with the starting point of the time period;

3. The numbers of accidents occurred in any nonoverlapping time periods are independent of one another;

4. In a sufficiently small time interval $\Delta t$ the probability of occurrence of one accident is proportional to $\Delta t$, and the probability of occurrence of above two accidents is higher order infinitesimal of $\Delta t-\mathrm{o}(\Delta t)$.

It is obvious that the first three properties above are all consistent with the facts of accidents. And the fourth property is also a reasonable assumption for construction accident. Therefore, it can be assumed that the occurrences of accidents, corresponding to the crossings of the risk process and safety threshold (Figure 1), are in accordance with Poisson process. That is, the total number of the crossings of $X(t)$ and the threshold $b n_{b}(t)$ in a given period of time $(0, T]$ is a Poisson process.

Generally, suppose process $X(t)$ be a non-stationary random process with a safety range of $-b_{2}, b_{1}$. The probability that total number $n_{b 1}^{+}(T)$ of $X(t)$ crossing the positive boundary $b_{1}$ with a positive slope in $(0, T]$ equals $i$ is

$$
P\left\{n_{b 1}^{+}=i\right\}=\frac{1}{i !}\left[\int_{0}^{T} v_{b 1}^{+}(\tau) d \tau\right]^{i} \exp \left[-\int_{o}^{T} v_{b 1}^{+}(\tau) d \tau\right],
$$

where $n_{b 1}^{+}(\tau)$ is the expectation of the number of the crossings of $X(t)$ and the positive boundary $b_{1}$ per unit time at time $\tau$, which is called crossing rate.

The probability that total number $v_{b 2}^{-}(T)$ of $X(t)$ crossing the negative boundary $-b_{2}$ with a negative slope in $(0, T]$ equals $i$ is

$$
P\left\{n_{b 2}^{-}=i\right\}=\frac{1}{i !}\left[\int_{o}^{T} v_{b 2}^{-}(\tau) d \tau\right]^{i} \exp \left[-\int_{o}^{T} v_{b 2}^{-}(\tau) d \tau\right],
$$

where $v_{b 2}^{-}(\tau)$ is the expectation of the number of the crossings of $X(t)$ and the negative boundary $-b_{2}$ per unit time at time $\tau$.

Obviously, the safety probability of $X(t)$ in $(0, T]$ is equal to the probability that $X(t)$ never exceeds the region $\left(b_{1},-b_{2}\right)$, i.e.

$$
P_{s 2}\left(b_{1},-b_{2}\right)=P\left\{\max X(t) \leq b_{1} \cap \min X(t) \geq-b_{2}, 0<t<T\right\} .
$$


Using Poisson crossing hypothesis (Zhang et al., 2019b), any two crossing events are independent of each other, then Eqn (3) can be further expressed as

$$
\begin{aligned}
& P_{s 2}\left(b_{1},-b_{2}\right)=P\left\{n_{b 1}^{+}(T)=0 \cap n_{b 2}^{-}(T)=0\right\}= \\
& P\left\{n_{b 1}^{+}(T)=0\right\} \cdot P\left\{n_{b 2}^{-}(T)=0\right\}= \\
& \exp \left[-\int_{0}^{T} v_{b 1}^{+}(\tau) d \tau\right] \cdot \exp \left[-\int_{0}^{T} v_{b 2}^{-}(\tau) d \tau\right]= \\
& \exp \left[-\int_{0}^{T}\left[v_{b 1}^{+}(\tau)+v_{b 2}^{-}(\tau)\right] d \tau\right] .
\end{aligned}
$$

Equation (4) is the calculation formula of the construction safety probability of random process $X(t)$ in period $(0, T]$ under Poisson assumption.

When the safety limit is symmetric two-sided boundary, i.e. $\left(b_{1}=b_{2}=b\right)$, Eqn (4) can be simplified as

$$
P_{s 2}\left(b_{1},-b_{2}\right)=\exp \left[-2 \int_{0}^{T} v_{b}(\tau) d \tau\right]
$$

where $v_{b}(\tau)=v_{b 2}^{-}(\tau)=v_{b 1}^{+}(\tau)$.

For the case of unilateral safety boundary, let $b_{1}=b$ and $b_{2} \rightarrow \infty$, then

$$
P_{s 2}\left(b_{1},-b_{2}\right)=\exp \left[-\int_{0}^{T} v_{b}^{+}(\tau) d \tau\right]
$$

If the random process $X(t)$ is stationary, the crossing rates $v_{b 1}^{+}(\tau)$ and $v_{b 2}^{-}(\tau)$ are independent of time, that is $v_{b 2}^{-}(\tau)=v_{b 2}^{-}, v_{b 1}^{+}(\tau)=v_{b 1}^{+}$, the corresponding Eqns (4)-(6) become

$$
\begin{aligned}
& P_{s 2}\left(b_{1},-b_{2}\right)=\exp \left[-\left(v_{b 1}^{+}+v_{b 2}^{-}\right) T\right] \\
& P_{s 2}(b,-b)=\exp \left(-2 v_{b} T\right) \\
& P_{s 1}(b)=\exp \left(-v_{b}^{+} T\right) .
\end{aligned}
$$

It can be seen from Eqns (4)-(9) that the crossing rates $v_{b 1}^{+}(\tau)$ and $v_{b 2}^{-}(\tau)$ is the key to calculate the construction safety probability.

\subsection{Calculation of the rate of crossing of risk-accident process and safety boundary}

It is the basis of safety probability calculation to study the crossing of the random risk-accident process and a certain safety boundary. The events of random risk-accident process $X(t)$ exceeding the threshold $b$ in a period of time $\left(t_{0}, t_{0}+T\right)$ can be expressed as follows:

$$
\bar{\theta}=\{X(t)>b, \forall t \in T\} .
$$

Suppose $X(t)\left(t \in T=\left(t_{0}, t_{0}+T\right)\right)$ is a mean square differentiable random process. Define a new random process as follows:

$$
Y(t)=\varepsilon[X(t)-b](t \in T),
$$

where $\varepsilon(\cdot)$ is unit step function. Therefore, the formal derivative of $Y(t)$ is

$$
\dot{Y}(t)=\dot{X}(t) \delta[X(t)-b](t \in T),
$$

where $\varepsilon(\cdot)$ is Dirac $-\delta$ function.
Random process $Y(t)$ is a $0-1$ process, i.e.

$$
Y(t)=\left\{\begin{array}{l}
1 X(t) \geq b \\
0 X(t)<b
\end{array} .\right.
$$

Therefore, $Y(t)$ can be regarded as a counting function, and every time $X(t)$ crosses the boundary $b$ with a positive or negative slope, the corresponding value is 1 or 0 . As a function of $t, \delta$ in Eqn (11) has weight function $1 / \dot{X}(t)$, so $\dot{X}(t)$ is a transcendence functional. Use a unit pulse to represent a transcendence event. The sign of the pulse depends on the sign of $\dot{X}(t)$.

So

$n_{b}(t)=\int_{t_{0}}^{t_{0}+T}|\dot{X}(t)| \delta[X(t)-b] d t$.

$n_{b}(t)$ is the total number of the crossings of $X(t)$ and the threshold $b$ in time period $T$, which is a random variable. So, the mathematical expectation of $n_{b}(t)$ can be obtained as

$$
\begin{aligned}
& N_{b}(t)=E\left[n_{b}(t)\right]=\int_{t_{0}}^{t_{0}+T} E\{|\dot{X}(t)| \delta[X(t)-b]\} d t= \\
& \int_{t_{0}}^{t_{0}+T} \int_{-\infty}^{\infty} \int_{-\infty}^{\infty}|\dot{X}| \delta(x-b) f_{x \dot{x}}(x, \dot{x}, t) d x d \dot{x} d t= \\
& \int_{t_{0}}^{t_{0}+T} \int_{-\infty}^{\infty}|\dot{x}| f_{x \dot{x}}(b, \dot{x}, t) d \dot{x} d t .
\end{aligned}
$$

$f_{x \dot{x}}(x, \dot{x}, t)$ is the joint probability density function of $X(t)$ and $\dot{X}(t)$.

In Eqn (14), let

$$
v_{b}(t)=\int_{-\infty}^{\infty}|\dot{x}| f_{x \dot{x}}(b, \dot{x}, t) d \dot{x} .
$$

Clearly, $v_{b}(t)$ is the mathematical expectation of the number of the crossing of $X(t)$ and the threshold $b$ per unit time at time $t(t \in T)$, that is the crossing rate.

If $X(t)$ is a stationary process, due to $f_{x \dot{x}}(x, \dot{x}, t)=$ $f_{x \dot{x}}(x, \dot{x})$, Eqn (15) becomes

$$
v_{b}(t)=\int_{-\infty}^{\infty}|\dot{x}| f_{x \dot{x}}(b, \dot{x}, t) d \dot{x}=v_{b} .
$$

So, the crossing rate of $X(t)$ is constant, which is independent of time.

\section{Construction safety accident process simulation based on Markov process}

\subsection{Markov characteristics of construction safety accident process}

Given the present state and all the past states of a stochastic process, the conditional probability distribution of its future state only depends on the present state. This type of stochastic process is Markov process. In other words, when the state of the process at time $t_{m}$ (present) is known, the probabilistic characteristics of the state of the process at time later than $t_{m}$ (future) will depend only on the state at time $t_{m}$, and be independent of the state at time earlier than $t_{m}$ (past). So, Markov process is without aftereffect for the past states. Non-aftereffect is an important property of Markov process. 
The non-aftereffect property is just the feature in the process of safety risk gradually evolving into safety accident in civil engineering construction (Winge et al., 2019). That is, in the process of safety risk event evolution, the status of a risk event at the present moment will affect the status of the risk event at the next moment, while the previous status of the risk event has no influence on the future status (the status after the present moment) of the risk event. According to the characteristics of the evolution process of safety risk event, it is obviously reasonable to adopt Markov process to simulate the development and evolution process of safety risk in civil engineering construction. The non-aftereffect property is a general characteristic of a safety risk-accident process.

When a random process $X(t)$ is adopted to simulate a safety risk-accident process, the occurrence of a safety accident corresponds to the random process $X(t)$ exceeding the safety threshold. Thus, the occurrence probability of a safety accident in a period of time $(0, T]$ is equivalent to the probability that the process $X(t)$ exceeding the safety threshold once within $(0, T]$.

When a random process $X(t)$ is adopted to simulate a safety risk-accident process, the occurrence of a safety accident corresponds to the random process $X(t)$ exceeding the safety threshold. Thus, the occurrence probability of a safety accident in a period of time $(0, T]$ is equivalent to the probability that the process $X(t)$ exceeding the safety threshold once within $(0, T]$.

Assuming that the safety threshold is $x=b$ and it is fixed and does not change with time (Figure 2). Then the construction safety probability $P_{s 1}(b)$ within the time period $(0, T]$ can be calculated by the following formula:

$$
P_{s 1}=P\{X(t) \leq b, 0<t \leq T\} .
$$

Equation (17) can be expressed as:

$$
P_{s 1}=P\{\max X(t) \leq b, 0<t \leq T\},
$$

where $\max X(t)$ is the maximum value of random process $X(t)$ within the time period $(0, T]$.

The time $T_{f 1}$ when the risk-accident process $X(t)$ first exceeds the safety threshold $(x=b)$ is a random variable, the probability distribution function $F_{T_{f 1}}(T)$ can be expressed as:

$$
F_{T_{f 1}}(t)=P\left\{T_{f 1} \leq t, 0<t \leq T\right\} .
$$

Obviously, the maximum value of $F_{T_{f 1}}(t)$ is $F_{T_{f 1}}(T)$, which is actually the failure probability. Therefore, the relation between $P_{s 1}(b)$ and $F_{T_{f 1}}(T)$ is as follows:

$$
P_{s 1}(b)=1-F_{T_{f 1}}(T) \text {. }
$$

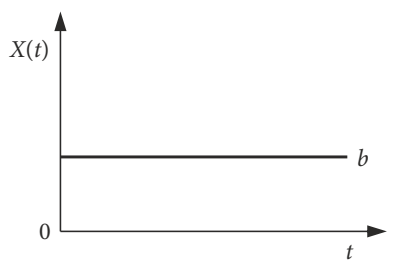

Figure 2. Fixed safety threshold
It shows that calculating the construction safety probability is equivalent to calculating the probability distribution function of the time when the risk process $X(t)$ first exceeds the safety threshold.

The following is to solve the first-passage problem of a continuous Markov process.

\subsection{The first passage probability of continuous Markov process}

The continuous Markov process can be determined by its initial conditions and the transition probability distribution function (or density). The transition probability distribution function plays a leading role in the probability distribution of the Markov process. Therefore, solving the first-passage problem of continuous Markov process is to establish the internal relation between the transition probability distribution function of Markov process and the first-passage time probability distribution (Yang \& Zhang, 2011). The relation between them can be used to solve the probability distribution of the first-passage time.

As shown in Figure 3, the transition probability from the state $X(0)=x_{0}$ of the process at time zero to the state $X(t) \leq b$ at time $t$ is $P\left\{X(t) \mid X(0)=x_{0}\right\}=F\left(b, t \mid x_{0}, 0\right)$. $F\left(b, t \mid x_{0}, 0\right)$ is a binary function of $b$ and $t$. It's easy to know that the derivative of $F\left(b, t \mid x_{0}, 0\right)$ with respect to time variable $t$ means the rate of change of probability $P\left\{X(t) \leq b \mid X(0)=x_{0}\right\}$ at time $t$ under the condition of $X(0)=x_{0}$.

Consider the small time increment $\Delta t$ at time $t$. The probability of $P\left\{X(t+\Delta t) \leq b \mid X(0)=x_{0}\right\}$ at time $t+\Delta t$ will also produces a smaller increment than the probability of $P\left\{X(t) \leq b \mid X(0)=x_{0}\right\}$ at time $t$. Then the difference between the probability at time $t+\Delta t$ and the probability at time $t$ (i.e., the change of probability),

$$
P\left\{X(t+\Delta t) \leq b \mid X(0)=x_{0}\right\}-P\left\{X(t) \leq b \mid X(0)=x_{0}\right\},
$$

is equivalent to the difference between the probability of the state $X(t) \leq b$ at time $t$ turning to the state $X(t+\Delta t)>b$ at time $t+\Delta t, P\{X(t+\Delta t)>b \mid X(t) \leq b\}$ and the probability of from the state $X(t)>b$ at time $t$ turning to the state $X(t+\Delta t) \leq b$ at time $t+\Delta t, P\{X(t+\Delta t) \leq b \mid X(t)>b\}$, $P\{X(t+\Delta t) \leq b \mid X(t)>b\}-P\{X(t+\Delta t)>b \mid X(t) \leq b\}$.

That is,

$$
\begin{aligned}
& P\left\{X(t+\Delta t) \leq b \mid X(0)=x_{0}\right\}-P\left\{X(t) \leq b \mid X(0)=x_{0}\right\}= \\
& P\{X(t+\Delta t) \leq b) \mid X(t)>b\}-P\{X(t+\Delta t)>b) \mid X(t) \leq b\} .
\end{aligned}
$$

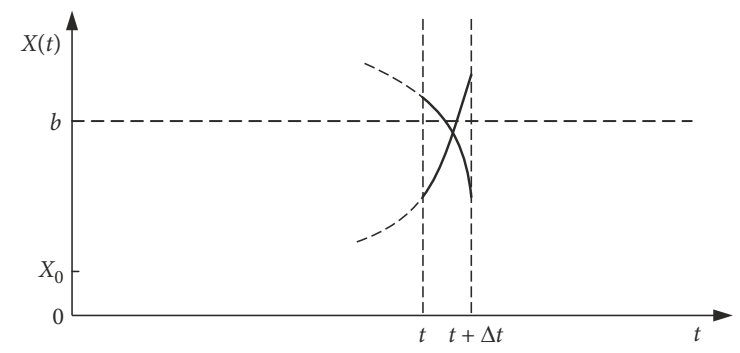

Figure 3 . The relationship between the transition probability distribution and the first-passage time distribution 
Equation (21) can be expressed using transition probability distribution function as below.

$$
\begin{aligned}
& F\left(b, t+\Delta t \mid x_{0}, 0\right)-F\left(b, t \mid x_{0}, 0\right)= \\
& P\{X(t+\Delta t) \leq b) \mid X(t)>b\}-P\{X(t+\Delta t)>b) \mid X(t) \leq b\} .
\end{aligned}
$$

Consider the term $P\{X(t+\Delta t)>b \mid X(t) \leq b\}$ on the right of Eqn (22). Because the time interval $\Delta t$ is sufficiently small, it can be considered that process $X(t)$ crosses the safety threshold $x=b$ at most once during $\Delta t$. This means that the probability of returning to state $X(\cdot) \leq b$ after $X(t)$ has crossed the threshold $x=b$ is zero (Figure 4 ). So, during $\Delta t$ if $X(t)$ goes beyond the threshold it has to go into interval $X(\cdot)>b$. On the other hand, according to the non-aftereffect property of Markov process, the state at time $t+\Delta t$ only depends on the state at time $t$. So, the probability $P\{X(t+\Delta t)>b \mid X(t) \leq b\}$ should be equal to the probability of the first passage in period $(t, t+\Delta t)$, namely,

$$
P\{X(t+\Delta t)>b) \mid X(t) \leq b\}=P\left\{t<T_{f} \leq t+\Delta t\right\}=f_{T_{f}} \Delta t .
$$

$f_{T_{f}}(t)$ is the probability density function of the first overtime $T_{f}$.

Then consider the term $P\{X(t+\Delta t) \leq b \mid X(t)>b\}$ on the left of Eqn (22). Regard $X(t+\Delta t)$ and $X(t)$ as twodimensional random vectors $(X(t+\Delta t), X(t))$. According to the conditional probability formula:

$$
P\{X(t+\Delta t) \leq b) \mid X(t)>b\}=\frac{P\{X(t+\Delta t) \leq b, X(t)>b\}}{P\{X(t)>b\}} .
$$

And the molecular term on the right side of Eqn (24) can be converted into (see Figure 5):

$$
\begin{aligned}
& P\{X(t+\Delta t) \leq b) \mid X(t)>b\}=P\{X(t+\Delta t) \leq b, X(t)<\infty\}- \\
& P\{X(t+\Delta t) \leq b), X(t) \leq b\}=P\{X(t+\Delta t) \leq b\}-[P\{X(t+\Delta t) \leq \\
& \infty, X(t) \leq b\}-P\{X(t+\Delta t)>b, X(t) \leq b\}]=P\{X(t+\Delta t) \leq b\}- \\
& P\{X(t) \leq b\}+P\{X(t+\Delta t)>b) \mid X(t) \leq b\} \cdot P\{X(t) \leq b\}= \\
& P\{X(t+\Delta t) \leq b\}-P\{X(t) \leq b\}+f_{T_{f}}(t) \Delta t \cdot P\{X(t) \leq b\} .
\end{aligned}
$$

The last equal sign in the above equation utilizes the result of Eqn (22). Then, substitute the result of the above equation into Eqn (24), and yields:

$$
\begin{aligned}
& P\{X(t+\Delta t) \leq b) \mid X(t)>b\}= \\
& \frac{P\{X(t+\Delta t) \leq b)\}-P\{X(t) \leq b\}+f_{T_{f}}(t) \Delta t \cdot P\{X(t) \leq b\}}{P\{X(t)>b\}} .
\end{aligned}
$$

By substituting Eqn (23) and Eqn (26) into Eqn (22) it can be obtained that:

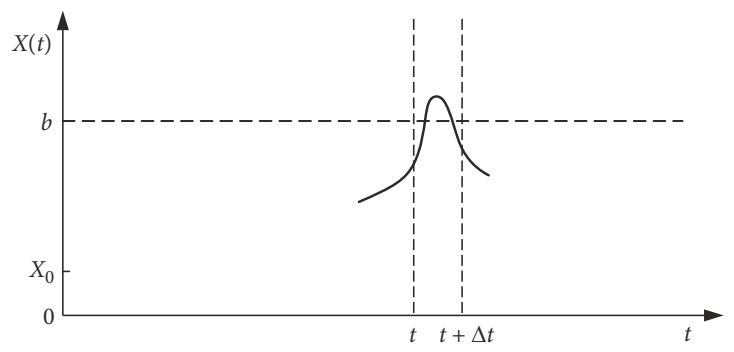

Figure 4 . The probability of returning after exceeding within $\Delta t$ is zero

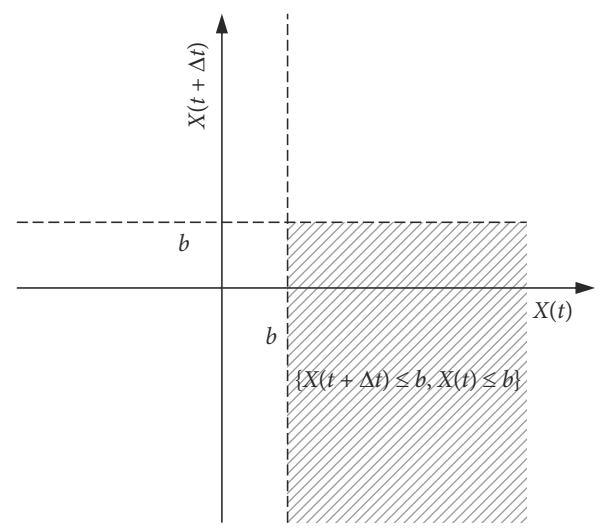

Figure 5. Probability Calculation based on the two-dimensional random vector $(X(t+\Delta t), X(t))$

$$
\begin{aligned}
& F\left(b, t+\Delta t \mid x_{0}, 0\right)-F\left(b, t \mid x_{0}, 0\right)= \\
& \frac{P\{X(t+\Delta t) \leq b)\}-P\{X(t) \leq b\}+f_{T_{f}}(t) \Delta t \cdot P\{X(t) \leq b\}}{P\{X(t)>b\}} \\
& f_{T_{f}}(t) \Delta t .
\end{aligned}
$$

Divide both sides of Eqn (27) by $\Delta t$, and then take the limit by $\Delta t \rightarrow 0$,

$$
\begin{aligned}
& \lim _{\Delta t \rightarrow 0} \frac{F\left(b, t+\Delta t \mid x_{0}, 0\right)-F\left(b, t \mid x_{0}, 0\right)}{\Delta t}= \\
& \lim _{\Delta t \rightarrow 0}\left[\frac{\frac{P\{X(t+\Delta t) \leq b)\}-P\{X(t) \leq b\}}{\Delta t}+f_{T_{f}}(t) P\{X(t) \leq b\}}{P\{X(t)>b\}}-f_{T_{f}}(t)\right]= \\
& \frac{\lim _{\Delta t \rightarrow 0} \frac{P\{X(t+\Delta t) \leq b)\}-P\{X(t) \leq b\}}{\Delta t}+f_{T_{f}}(t) P\{X(t) \leq b\}}{P\{X(t)>b\}}-f_{T_{f}}(t) .
\end{aligned}
$$

According to the definition of derivative, it is noted that:

$$
\begin{aligned}
& \lim _{\Delta t \rightarrow 0} \frac{F\left(b, t+\Delta t \mid x_{0}, 0\right)-F\left(b, t \mid x_{0}, 0\right)}{\Delta t}=\frac{\partial}{\partial t} F\left(b, t \mid x_{0}, 0\right) ; \\
& \lim _{\Delta t \rightarrow 0} \frac{P\{X(t+\Delta t) \leq b\}-P\{X(t) \leq b\}}{\Delta t}= \\
& \lim _{\Delta t \rightarrow 0} \frac{F(b, t+\Delta t)-F(b, t)}{\Delta t}=\frac{\partial}{\partial t} F(b, t) .
\end{aligned}
$$


Thus, Eqn (28) can be expressed as:

$$
\frac{\partial}{\partial t} F\left(b, t \mid x_{0}, 0\right)=\frac{\frac{\partial}{\partial t} F(b, t)+f_{T_{f}}(t) P\{X(t) \leq b\}}{P\{X(t)>b\}}-f_{T_{f}}(t) .
$$

Equation (29) shows the relation between the probability density of first-passage time and the transition probability distribution function of Markov process. From the formula, the analytical expression of the probability density function $f_{T_{f}}(t)$ of the first passage time $T_{f}$ can be conveniently solved as follows:

$$
f_{T_{f}}(t)=\frac{F_{t}^{\prime}\left(b, t \mid x_{0}, 0\right) \cdot[1-F(b, t)]-F_{t}^{\prime}(b, t)}{2 F(b, t)-1},
$$

where $F_{t}^{\prime}\left(b, t \mid x_{0}, 0\right)=\frac{\partial}{\partial t} F\left(b, t \mid x_{0}, 0\right)$ is the partial derivative of the transition probability distribution function of Markov process with respect to $t$ under the condition of $x_{0}, F(b, t)$ is the one-dimensional distribution function $\left.F(x, t)\right|_{x=b}$ of Markov process, $F_{t}^{\prime}(b, t)$ is the partial derivative of the one-dimensional distribution function with respect to $t,\left.F_{t}^{\prime}(x, t)\right|_{x=b}$.

\subsection{Safety probability during construction period based on continuous Markov process simulation}

Suppose the safety threshold is $x=b$, and the length of time period to be examined is $T$. It is easy to know that the time $T_{f}$ when the random process $X(t)$ first exceeds the safety threshold in the time period $(0, T]$ is a continuous random variable. So the probability distribution function is:

$$
F_{T_{f}}(t)=P\left\{T_{f} \leq t, 0<t \leq T\right\} .
$$

Obviously, the maximum value of $F_{T_{f}}(t)$ is $F_{T_{f}}(T)$ when $t=T$. According to Eqn (31) it can be obtained that:

$$
F_{T_{f}}(t)=P\left\{T_{f} \leq t\right\} .
$$

Equation (32) indicates that $F_{T_{f}}(T)$ represents the probability that the first passage time $T_{f}$ is within the time period $(0, T]$, so it is actually the failure probability. Therefore, the construction safety probability $P_{s}(b)$ and $F_{T_{f}}(T)$ have the following relationship:

$$
P_{s}(b)=1-F_{T_{f}}(T) \text {. }
$$

That is, the reliability can be obtained from the probability distribution function of the first passage time.

After obtaining the probability density function $f_{T_{f}}(t)$ of the first-passage time of the continuous Markov process from Eqn (30), the construction safety probability $P_{s}(b)$ in the period of $(0, T]$ can be calculated according to the relationship between $f_{T_{f}}(t)$ and the probability distribution function of the first-passage time.

$$
P_{s}(b)=1-\int_{0}^{T} f_{T_{f}}(t) d t .
$$

\subsection{Application example}

Assuming that a construction risk process is Markov process $X(t)$, the transition probability density function is

$$
f\left(x, t \mid x_{0}, t_{0}\right)=\frac{x-x_{0}}{\sigma^{2}\left(t-t_{0}\right)} \exp \left[\frac{\left(x-x_{0}\right)^{2}}{2 \sigma^{2}\left(t-t_{0}\right)}\right],
$$

where $t \geq 0$, and $\sigma=1$ is distribution parameter. The initial condition is

$$
f(x, 0 \mid 0,0)=\delta(x) .
$$

The mathematical expectation and variance of the random process $X(t)$ are

$$
E[X(t)]=\sqrt{\frac{\pi}{2}} \sigma t^{\frac{1}{4}}, D[X(t)]=\frac{4-\pi}{2} \sigma^{2} t .
$$

According to Eqn (30), $f_{T_{f}}(t)$ can be obtained as:

$$
f_{T_{f}}(t)=\frac{\left[\exp \left(-\frac{b}{2 \sigma^{2} t}\right)-1\right] \frac{b}{2 \sigma^{2} t} \exp \left(-\frac{b}{2 \sigma^{2} t}\right)}{2 \exp \left(-\frac{b}{2 \sigma^{2} t}\right)-1} .
$$

To verify the correctness of the conclusion in detail, the results are discussed in two cases of infinite and finite.

1) Infinite threshold situation

Consider the case of infinite threshold. Order $b \rightarrow \infty$ and solve the limit of Eqn (38). According to the limit algorithm and 1 'hopital's rule it can be gotten that

$\lim _{b \rightarrow \infty} f_{T_{f}}(t)=0$.

Therefore,

$$
P_{f}=\int_{0}^{T} f_{T_{f}}(t) d t=0
$$

Equation (40) indicates that when the safety limit approaches infinity, the probability of safety accidents is zero, which is consistent with reality.

2) Finite threshold situation

When the safety threshold is finite, the occurrence probability of construction accident is solved according to the integral $\int_{0}^{T} f_{T_{f}}(t) d t$. Several increasing period lengths are selected for calculation based on the safety threshold $b=10$, as shown in Figure 6. It can be seemed that the probability of accidents increases with time. This is consistent with the reality.

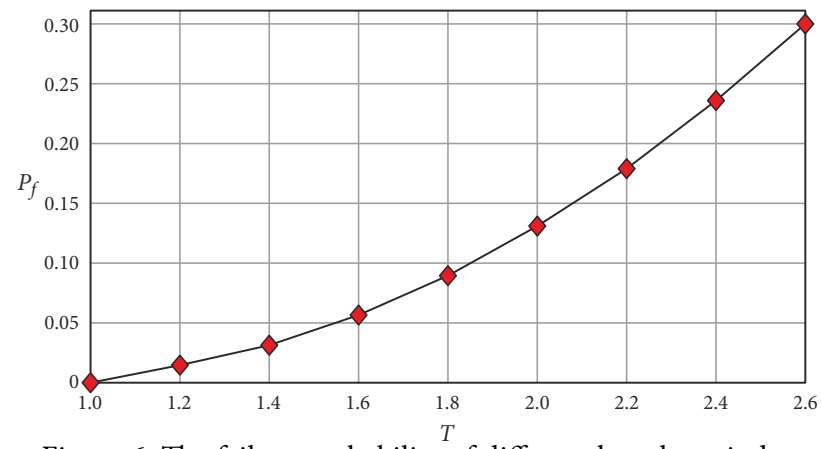

Figure 6 . The failure probability of different length periods in finite threshold case 


\section{Construction safety risk-accident process simulation based on normal random process}

\subsection{Normal characteristics of construction safety risk-accident process}

Some types of construction accidents are generated by the combination and development of multiple risk factors. In multiple risk factors, each risk factor is irrelevant to each other and does not play a leading role. Such construction risk-accident process can be simulated by normal stochastic process.

If arbitrary $n$-dimensional random vector $\left\{Z_{1}(t), Z_{2}(t)\right.$ $\left.\cdots Z_{n}(t)\right\}$ of random process $\{X(t), t \in T\}$ is $n$-dimensional normal random vector, the process is normal process. Its probability density function is

$$
\begin{aligned}
& f\left(Z_{1}, Z_{2}, Z_{3}, \cdots Z_{n}, t_{1}, t_{2}, t_{3} \cdots t_{n}=\right. \\
& \frac{1}{2 \pi^{\frac{n}{2}}|C|^{\frac{1}{2}}} \exp \left\{-\frac{1}{2}(z-m(t))^{T} C^{-1}(z-m(t))\right\},
\end{aligned}
$$

where:

$Z=\left[\begin{array}{c}Z_{1} \\ Z_{2} \\ \cdot \\ \cdot \\ Z_{n}\end{array}\right], m(t)=\left[\begin{array}{c}m_{Z_{1}}(t) \\ m_{Z_{2}}(t) \\ \cdot \\ \cdot \\ m_{Z_{n}}(t)\end{array}\right] ; C=\left[\begin{array}{cccc}K_{11} & K_{12} & \cdot & K_{1 N} \\ K_{21} & K_{22} & \cdot & K_{2 N} \\ \cdot & \cdot & \cdot & \cdot \\ \cdot & \cdot & \cdot & K_{N-1, N} \\ K_{N 1} & K_{N 2} & \cdot & K_{N N}\end{array} \mid=\right.$

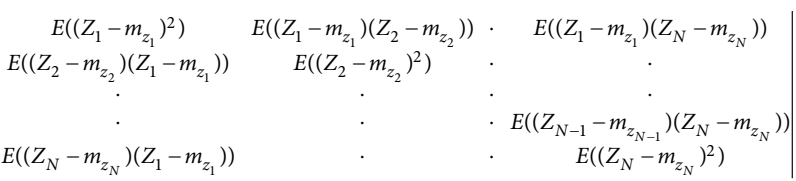

If $Z_{1}(t), Z_{2}(t), Z_{3}(t) \cdots Z_{n}(t)$ are independent of each other, then

$$
C=\left(\begin{array}{cccc}
\sigma_{11}^{2} & \cdot & \cdot & 0 \\
\cdot & \sigma_{22}^{2} & \cdot & \cdot \\
\cdot & \cdot & \cdot & \cdot \\
0 & \cdot & 0 & \sigma_{n n}^{2}
\end{array}\right)
$$

Thus the probability density function is

$$
\begin{aligned}
& f\left(Z_{1}, Z_{2}, Z_{3}, \cdots Z_{n}, t_{1}, t_{2}, t_{3} \cdots t_{n}\right)= \\
& \prod_{i=1}^{n} \frac{1}{\sqrt{2 \pi} \sigma_{i}\left(t_{1}\right)} \exp \left\{-\frac{\left(z_{1}-m_{i}\left(t_{i}\right)\right)^{2}}{2\left[\sigma_{i}\left(t_{i}\right)\right]^{2}}\right\} .
\end{aligned}
$$

\subsection{Analysis of construction safety probability based on normal process simulation}

When the risk-accident process $X(t)$ is a stationary normal process with zero mean value, the rate of the crossing of the process and the safety threshold can be obtained by Eqn (16):

$$
v_{b}^{+}=v_{b}^{-}=\frac{\sigma_{\dot{x}}}{2 \pi \sigma_{x}} \exp \left(-\frac{b^{2}}{2 \sigma_{x}^{2}}\right) .
$$

Substituting Eqn (44) into Eqn (7), the calculation formula of construction safety probability in the time period $(0, T]$ is obtained as follows:

$$
P_{s}\left(b_{1},-b_{2}\right)=\exp \left\{-\frac{\sigma_{\dot{x}} T}{2 \pi \sigma_{x}}\left[\exp \left(-\frac{b_{1}^{2}}{2 \sigma_{x}^{2}}\right)+\exp \left(-\frac{b_{2}^{2}}{2 \sigma_{x}^{2}}\right)\right]\right\} .
$$

Under the condition of unilateral safety threshold, Eqn (45) is simplified as:

$$
P_{s}(b)=\exp \left[-\frac{\sigma_{\dot{x}} T}{2 \pi \sigma_{x}} \exp \left(-\frac{b^{2}}{2 \sigma_{x}^{2}}\right)\right] .
$$

If the risk-accident process $X(t)$ is a nonstationary normal process with zero mean value, the crossing rate becomes

$$
\begin{aligned}
& v_{b 1}^{+}(t)=-\frac{\sigma_{\dot{x}} T}{2 \pi \sigma_{x}} \exp \left[-\frac{b_{1}^{2}}{2 \sigma_{x}^{2}(t)}\right] ; \\
& v_{b 2}^{-}(t)=-\frac{\sigma_{\dot{x}} T}{2 \pi \sigma_{x}} \exp \left[-\frac{b_{2}^{2}}{2 \sigma_{x}^{2}(t)}\right],
\end{aligned}
$$

where $\sigma_{x}(t)$ is the root mean square of the normal process at time $t$.

Substitute the crossing rate into Eqn (4), and yields the calculation formula of safety probability of the riskaccident process $X(t)$ in the time period $(0, T]$.

$$
\begin{aligned}
& P_{s}\left(b_{1},-b_{2}\right)= \\
& \exp \left\{-\frac{1}{2 \pi} \int_{0}^{T} \frac{\sigma_{\dot{x}}(t)}{\sigma_{x}(t)} \exp \left[-\frac{b_{1}^{2}}{2 \sigma_{x}^{2}(t)}-\frac{b_{2}^{2}}{2 \sigma_{x}^{2}(t)}\right] d t\right\} .
\end{aligned}
$$

If the number of the crossing of the process and the safety boundary conforms to Markov process, the calculation formula of construction safety probability of the process $X(t)$ in the time period $(0, T]$ can be concluded from Eqn (17).

$$
P_{s}(b,-b)=\exp \left[-\int_{0}^{T} \alpha(t) d t\right] \quad\left(b_{1}=b_{2}=b\right),
$$

where $\alpha(t), r(t)$ and $\omega_{2}(t)$ are respectively

$$
\begin{aligned}
& \alpha(t)=\frac{w_{2}(t)}{\pi} \exp \left[-\frac{r^{2}(t)}{2}\right] \frac{1-\exp \left[-\sqrt{\frac{\pi}{2}} q(t) r(t)\right]}{1-\exp \left[-\frac{r^{2}(t)}{2}\right]} ; \\
& r(t)=\frac{b}{\sigma_{x}(t)} ; w_{2}(t)=\frac{\sigma_{\dot{x}}(t)}{\sigma_{x}(t)} .
\end{aligned}
$$

The probability distribution of a normal process can be completely determined by mathematical expectation and covariance functions. Therefore, first, it is necessary to obtain the sample data of $n$-dimensional random vector $\left\{Z_{1}(t), Z_{2}(t), Z_{3}(t) \cdots Z_{n}(t)\right\}$ of the normal process through observation and record in past practical engineering, then the mean value and covariance of joint probability density function can be calculated by mathematical statistics method. That can provide a reference for the risk assessment and prediction of similar construction risk-accident process in the future. 


\section{Analysis of macroscopic statistical law of construction accidents}

\subsection{Poisson process simulation}

For practical engineering problems simplifying assumptions need to be made to establish theoretical model and make problem easy. Therefore, to explore the regularity of accidents in civil engineering construction should focus on the main characteristics of the construction phenomenon and discard the secondary factors to establish the model. This section makes statistics of different kinds of safety accidents in civil engineering construction site and analysis the regulation from a macroscopic perspective (Forteza et al., 2016; Zhang et al., 2020). Obviously, on the macro level, the number of accidents on the construction site is a counting process. According to the independent characteristics of construction accidents, it is reasonable to directly assume that the counting process is a Poisson process. Based on Poisson process, the total number of safety accidents during the whole construction period can be predicted.

Assuming that the state of random process $\{X(t), t \geq 0\}$ take only non-negative integers, if it satisfies (Ross, 1996):

1. $N(0)=0$;

2. $\{N(t), t \geq 0\}$ has independent increment;

3. For any $s, t>0$,

$P\{N(s+t)-N(s)=k\}=$

$\frac{1}{k !}\left[\int_{0}^{t} \lambda(\tau) d \tau\right]^{k} \exp \left[-\int_{0}^{t} \lambda(\tau) d \tau\right], k=0,1,2, \ldots ., \lambda>0$.

Then $\{N(t), t \geq 0\}$ is called Poisson process. Parameter $\lambda(\tau)$ represents the mathematical expectation of the number of random points per unit time at time $\tau$, and this kind of Poisson process is called inhomogeneous Poisson process. If $\lambda(\tau)=\lambda$ is constant and independent of time $\tau$, this kind of Poisson process is called the homogeneous Poisson process. In this case, Eqn (50) changes to the following equation:

$$
\begin{aligned}
& P\{N(s+t)-N(s)=k\}=P\{N(t)=k\}= \\
& \frac{(\lambda t)^{k}}{k !} e^{-\lambda t}, k=0,1,2 \ldots, \lambda>0 .
\end{aligned}
$$

Poisson process is a time continuous but state discrete random process. $N(t)$ represents the number of random points appear in the time period of $[0, t]$. Corresponding to the construction accidents, $N(t)$ represents the number of construction accidents in the whole construction period (denote construction period as $T$, then $t \in[0, T]$ ).

\subsection{The probability distributions of the time interval between adjacent accidents and the waiting time}

$T_{1}$ represents the time of the first accident. For $n>1, T_{n}$ represents the interval time between the $(n-1)$-th accident and the nth accident. Sequence $\left\{T_{n}, n=1,2,3, \ldots\right\}$ is the time series between accidents.
Now solve the distribution of $T_{n}$. Notice that the event $\left\{T_{1}>t\right\}$ happens only if no accident happens in the interval $[0, t]$. Therefore,

$$
P\left\{T_{1}>t\right\}=P\{N(t)=0\}=e^{-\lambda t} .
$$

So, $T_{1}$ follows an exponential distribution with the mean of $\lambda$. Then,

$$
P\left\{T_{2}>t\right\}=E\left[P\left\{T_{2}>t \mid T_{1}\right\}\right] .
$$

However,

$P\left\{T_{2}>t \mid T_{1}=s\right\}=P\left\{\right.$ zero accident in $\left.(s, s+t) \mid T_{1}=s\right\}=$

$P\{$ zero accident in $(s, s+t)\}=e^{-\lambda t}$,

which is obtained using the independent increment property and stationary increment property. Therefore, it can be seen that $T_{2}$ also follows an exponential distribution with the mean of $\lambda$, and $T_{2}$ is independent of $T_{1}$. Thus, by repeating the above steps it can be concluded that, $T_{n}(n=$ $1,2,3, \ldots)$ is an exponential random variable that is independently and identically distributed with a mean of $\lambda$. So, the probability density function of $T_{n}$ is

$$
f_{T_{n}}(t)=\lambda e^{-\lambda t}, t>0 .
$$

The above conclusion shows that the state of Poisson process is independent of everything that happened before (i.e. independent increment) and has the same distribution as the original process (i.e. stationary increment). These two characteristics also coincide with the characteristics of construction accident events. That is, the construction process is memoryless, construction accidents are independent of each other, an accident is not associated with another one. So, the exponential interval is exactly what is expected.

The time when the $n$th accident happens is denoted as $S_{n}$, and it is also called the waiting time for the $n$th accident. To solve the distribution of $S_{n}$, notice that the $n$th accident occurs at time $t$ only if the number of accidents until $t$ is at least $n$, that is

$$
N(t) \geq n \leftrightarrow S_{n} \leq t .
$$

Therefore,

$$
F_{S_{n}}(t)=P\left\{S_{n} \leq t\right\}=P\{N(t) \geq n\}=\sum_{j=n}^{\infty} e^{-\lambda t} \frac{(\lambda t)^{j}}{j !} .
$$

The above equation is differentiated and yields

$$
\begin{aligned}
& f_{S_{n}}(t)=-\sum_{j=n}^{\infty} \lambda e^{-\lambda t} \frac{(\lambda t)^{j}}{j !}+\sum_{j=n}^{\infty} \lambda e^{-\lambda t} \frac{(\lambda t)^{j-1}}{(j-1) !}= \\
& \lambda e^{-\lambda t} \frac{(\lambda t)^{n-1}}{(n-1) !}+\sum_{j=n+1}^{\infty} \lambda e^{-\lambda t} \frac{(\lambda t)^{j-1}}{(j-1) !}-\sum_{j=n}^{\infty} \lambda e^{-\lambda t} \frac{(\lambda t)^{j}}{(j-1) !}= \\
& \lambda e^{-\lambda t} \frac{(\lambda t)^{n-1}}{(n-1) !} .
\end{aligned}
$$

Thus, it can be concluded that the random variable $S_{n}$ follows the gamma distribution with parameters $n$ and $\lambda$, i.e.

$$
f_{S_{n}}(t)=\left\{\begin{array}{lc}
\lambda e^{-\lambda t} \frac{(\lambda t)^{n-1}}{(n-1) !} & t \geq 0 \\
0 & t<0
\end{array} .\right.
$$


Using Poisson process to predict the total number of safety accidents during the whole construction period, the key is to determine the parameter $\lambda$ of the Poisson process. $\lambda$ represents the average strength of construction accidents. Therefore, to determine its value, a large number of construction site safety accident investigation and statistics should be conducted. Then substitute the average intensity of construction accidents obtained from previous construction site statistics into the Poisson process model to predict the situation of safety accidents in similar construction site.

\section{Discussion and example}

This work first made the connection of stochastic process model to the construction safety accident conceptually, then derived the formulas of calculating the stochastic processes exceeding safety threshold, which is the key to judge whether a risk factor would evolve into an accident. And thus, stochastic process-based construction safety risk prediction and management theory framework was established. This work is important and necessary because it can reveal the dynamic stochastic law from risk to accident. Specifically, general process, Markov process, normal process and Poisson process were used to simulate future risk-accident process, for the purpose of prediction and management of safety risk. It should be pointed out that, to determine the parameters of the stochastic process model proposed, the corresponding data of previous construction accidents are needed. And in general, the more date, the more accurate the model.

Take the Poisson process model regarding the macroscopic statistical law of construction accidents presented in Section 5 as an example. Table 1 shows the statistical data of collapse accidents in China during 2004-2013.

The average number of collapse accidents is 103 per year. This means that under the current economic, technical and management level the intensity of such accident per unit time is 103 times a year. Parameter $\lambda$ represents the mathematical expectation of the number of random accidents per unit time, so parameter $\lambda=103$. According to Eqn (51):

$$
P\{N(t)=k\}=\frac{(103 t)^{k}}{k !} e^{-103 t}, k=0,1,2 \ldots
$$

According to Poisson process theory, $P\{N(t)=k\}$ means the probability of $k$ accidents in period of $(0, t)$. Thus, we can use this equation to predict the occurrence of accidents. For instance, let $t=1$ and $k=100$,

$$
P\{N(1)=100\}=\frac{(103 \times 1)^{100}}{100 !} e^{-103}=3.81 \% .
$$

This means the probability of 100 accidents in the period of $(0,1)$ (1 year) is $3.81 \%$. We can also use Eqn (59) to predict the accident probability in another period. For instance, let $t=0.5$ and $k=50$,

$$
P\{N(0.5)=50\}=\frac{(103 \times 0.5)^{500}}{50 !} e^{-103 \times 0.5}=5.51 \% .
$$

It means that the probability of 50 accidents in the period of $(0,0.5)$ (half a year) is $5.51 \%$. More calculation results are shown in Figures 7 and 8.

Further, we can get more accident prediction information according to Eqns (55) and (58). According to Eqn (55), the probability density function of the time interval between adjacent accidents is

$$
f_{T_{n}}(t)=103 e^{-103 t}(t>0) .
$$

Then, we can make the probabilistic prediction, for instance,

$$
P\left\{0.1<T_{n}<0.2\right\}=\int_{0.1}^{0.2} 103 e^{-103 t} d t=3.36 \times 10^{-5} .
$$

It means the time interval of two adjacent accidents is $0.1 \sim 0.2$ year (i.e., $36.5 \sim 73$ days) with the probability of $3.36 \times 10^{-5}$. We can decrease the time interval to obtain more accurate probability prediction, for example, change $(0.1,0.2)$ to $(0.1,0.15)$.

Using Eqn (58) we can predict by probability the occurrence time of the $n$-th accident. For example, the probability density function of the time when the second accident happens $S_{2}$ is

$$
f_{S_{2}}(t)= \begin{cases}103 e^{-103 t}(103 t) \quad t \geq 0 \\ 0 \quad t<0\end{cases}
$$

Then,

$$
F_{S_{2}}(t)=P\left\{S_{2}<t\right\}=\int_{0}^{t} f_{S_{2}}(u) d u=1-e^{-103 t}(1+103 t),
$$

which is the probability of two accidents occurring in the period $(0, t)$. For instance, let $t=0.03$,

$$
F_{S_{2}}(0.03)=1-e^{-103 \times 0.03}(1+103 \times 0.03)=0.81 \text {. }
$$

This indicates that the probability of two accidents occurring before 0.03 year ( 11 days) is 0.81 . More calculation results are shown in Figure 9. It can be seemed that the probability of the second accident occurring increases with time period. Especially, when $t$ reaches 0.08 , $F_{S_{2}}(0.08)$ is close to 0.99 , which indicates that the probability of the second accident occurring before 0.08 year (29.2 days) is very large. Actually, this result is very meaningful. It enlightens us that under the original situations

\begin{tabular}{|c|c|c|c|c|c|c|c|c|c|c|c|}
\hline Year & 2004 & 2005 & 2006 & 2007 & 2008 & 2009 & 2010 & 2011 & 2012 & 2013 & average \\
\hline Number of the accidents & 125 & 133 & 129 & 117 & 106 & 97 & 93 & 86 & 57 & 90 & 103 \\
\hline
\end{tabular}
(including construction environment, safety management, safety technology, etc.) it's almost certain that a second accident would happen before the 29th day (from the day when the prediction is made on). So, if the managers pay more attention to this problem based on the prediction result and strengthen safety management or strengthen

Table 1. Number of collapse accidents in China from 2004 to 2013 
the investigation of potential risk sources, the probability of potential accident might be greatly reduced.

Based on Poisson process model, the occurrence probability of $k$ accidents in period of $(0, t)$, the probabilistic prediction of the time interval between adjacent accidents and the probabilistic prediction of the occurrence time of the $n$-th accident can provide useful information of accidents to the project manager or the industry management government department in a region. In addition, it should be noted that the application of Markov process and normal process need much more detailed data of construction accidents. So, we need to make detailed statistics and records from now on.

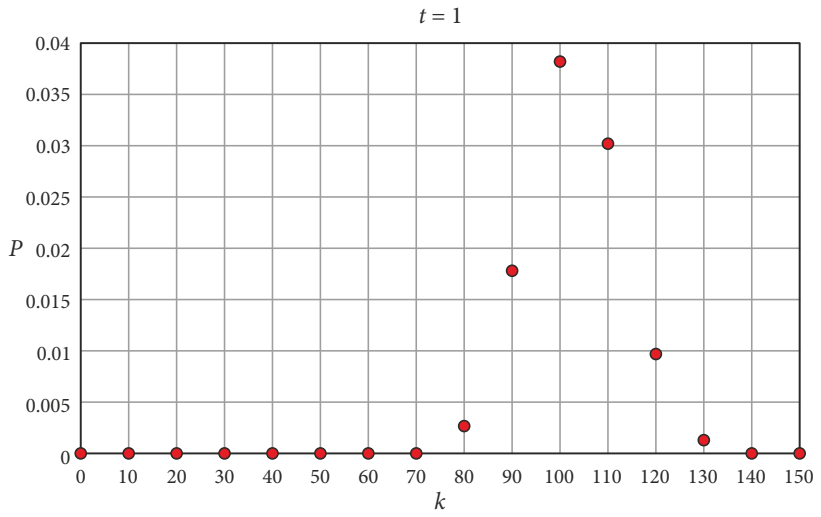

Figure 7. The probabilities of occurrence number of accidents in a period of 1 year

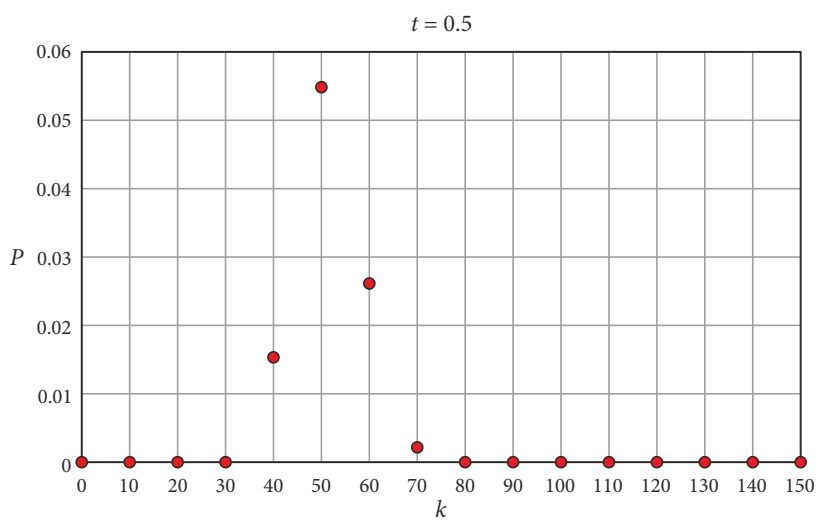

Figure 8 . The probabilities of occurrence number of accidents in a period of half a year

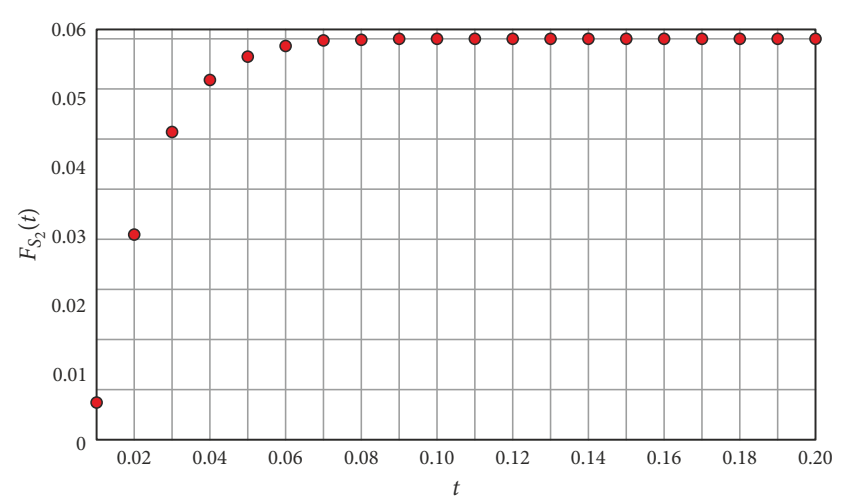

Figure 9. The probabilities of the second accident occurring before $t$ year

\section{Conclusions}

1. The occurrence and evolution process of construction safety risk can be regarded as a random process. The random process exceeding the safety threshold corresponds to safety accidents happening. Firstly, the construction risk-accident process is regarded as a general random process, then the probability of accidents in a certain period is calculated and the corresponding formulas are derived.

2. The non-aftereffect of Markov process is just the feature of the process of safety risk gradually evolving into an accident in civil engineering construction. According to Markov property, the development and evolution process of construction safety risk can be modeled and corresponding warning measures can be established to avoid the occurrence of construction accidents. And the expression of probability density function for the first-passage time of Markov process is derived to calculate the probability of accidents.

3. Among the various risk factors leading to construction accident, if each risk factor is irrelevant and does not play a leading role, such construction risk-accident process can be simulated by normal process. The construction safety probability formulas of stationary normal process and non-stationary normal process with zero mean value are derived respectively. These provide a reference for the risk assessment and prediction of the similar type of construction risk-accident process in the future.

4. Different kinds of safety accidents occurring on the construction site of civil engineering are analyzed from a macro perspective. The total number of safety accidents in the whole construction period can be predicted based on Poisson process. Moreover, the interval time between the $(n-1)$-th accident and the nth accident and the waiting time for the nth accident are obtained. These will play a macro guiding role in the prediction of construction accidents.

\section{Funding}

This was supported by the $<$ Research Project of the Educational Commission of Hunan Province> under Grant [number 19B020]; < National Natural Science Foundation of China> under Grant [number 51678067, 51808054]; $<$ Systematic Project of Guangxi Key Laboratory of Disaster Prevention and Structural Safety> under Grant [number 2016ZDK014].

\section{Author contributions}

Zhenhao Zhang conceived the study and was responsible for the design and development of the data analysis. Wenbiao $\mathrm{Li}$ was responsible for data collection and analysis. Jianyu Yang was responsible for data interpretation. 


\section{Disclosure statement}

All the authors have no conflict of interest.

\section{References}

Amin, M., Imtiaz, S., \& Khan, F. (2018). Process system fault detection and diagnosis using a hybrid technique. Chemical Engineering Science, 189(2), 191-211. https://doi.org/10.1016/j.ces.2018.05.045

Amin, M., Khan, F., \& Imtiaz, S. (2019). Fault detection and pathway analysis using a dynamic Bayesian network. Chemical Engineering Science, 195(23), 777-790.

https://doi.org/10.1016/j.ces.2018.10.024

Andolfo, C., \& Sadeghpour, F. (2015). A probabilistic accident prediction model for construction sites. Procedia Engineering, 123, 15-23. https://doi.org/10.1016/j.proeng.2015.10.052

Ang, A. H.-S., \& Tang, W. H. (2007). Probability concepts in engineering - emphasis on applications to civil and environmental engineering. John Wiley \& Sons Ltd.

Arunthavanathan, R., Khan, F., Ahmed, S., Imtiaz, S., \& Rusli, R. (2020). Fault detection and diagnosis in process system using artificial intelligence-based cognitive technique. Computers \& Chemical Engineering, 134(4), 106697.

https://doi.org/10.1016/j.compchemeng.2019.106697

Ayhan, B. U., \& Tokdemir, O. B. (2019). Predicting the outcome of construction incidents. Safety Science, 113, 91-104. https://doi.org/10.1016/j.ssci.2018.11.001

Bhatia, K., Khan, F., Patel, H., \& Abbassi, R. (2019). Dynamic risk-based inspection methodology. Journal of Loss Prevention in the Process Industries, 62, 103974.

https://doi.org/10.1016/j.jlp.2019.103974

Chen, C.-W., Leu, S-S., LiN, C-C., \& Fan, C. (2010). Characteristic analysis of occupational accidents at small construction enterprises. Safety Science, 48(6), 698-707.

https://doi.org/10.1016/j.ssci.2010.02.001

Chi, S., \& Han, S. (2013). Analyses of systems theory for construction accident prevention with specific reference to OSHA accident reports. International Journal of Project Management, 31(7), 1027-1041.

https://doi.org/10.1016/j.ijproman.2012.12.004

Choe, S., \& Leite, F. (2020). Transforming inherent safety risk in the construction Industry: A safety risk generation and control model. Safety Science, 124, 104-594.

https://doi.org/10.1016/j.ssci.2019.104594

de Lamos, T., Eaton, D., Betts, M., \& de Almeida, L. T. (2004). Risk management in the Lusoponte concession-a case study of the two bridges in Lisbon, Portugal. International Journal of Project Management, 22(1), 63-73. https://doi.org/10.1016/S0263-7863(03)00013-9

Ding, L., Ji, J., \& Khan, F. (2020). Combining uncertainty reasoning and deterministic modeling for risk analysis of fireinduced domino effects. Safety Science, 129, 104802. https://doi.org/10.1016/j.ssci.2020.104802

Don, M. G., \& Khan, F. (2019). Dynamic process fault detection and diagnosis based on a combined approach of hidden Markov and Bayesian network model. Chemical Engineering Science, 201, 82-96. https://doi.org/10.1016/j.ces.2019.01.060

Faber, M. H. (2003). Risk and safety in civil, surveying and environmental engineering. Swiss Federal Institute of Technology.

Forteza, F. J., Sesé, A., \& Carretero-Gómez, J. M. (2016). CONSRAT. Construction sites risk assessment tool. Safety Science, 89, 338-354. https://doi.org/10.1016/j.ssci.2016.07.012
Golizadeh, H., Hon, C. K. H., Drogemuller, R., \& Hosseini, M. R. (2018). Digital engineering potential in addressing causes of construction accidents. Automation in Construction, 95, 284-295. https://doi.org/10.1016/j.autcon.2018.08.013

Hassan, J., \& Khan, F. (2012). Risk-based asset integrity indicators. Journal of Loss Prevention in the Process Industries, 25(3), 544-554. https://doi.org/10.1016/j.jlp.2011.12.011

Hoła, B., \& Szóstak, M. (2014). Analysis of the development of accident situations in the construction industry. Procedia Engineering, 91, 429-434.

https://doi.org/10.1016/j.proen.2014.12.0488

Irumba, R. (2014). Spatial analysis of construction accidents in Kampala, Uganda. Safety Science, 64, 109-120. https://doi.org/10.1016/j.ssci.2013.11.024

Isaac, S., \& Edrei, T. (2016). A statistical model for dynamic safety risk control on construction sites. Automation in Construction, 63, 66-78. https://doi.org/10.1016/j.autcon.2015.12.006

Jamot, D. G. C, \& Park, J. Y. (2019). System theory based hazard analysis for construction site safety: A case study from Cameroon. Safety Science, 118, 783-794. https://doi.org/10.1016/j.ssci.2019.06.007

Jin, R., Wang, F., \& Liu, D. (2020). Dynamic probabilistic analysis of accidents in construction projects by combining precursor data and expert judgments. Advanced Engineering Informatics, 44, 101062. https://doi.org/10.1016/j.aei.2020.101062

Kang, K., \& Ryu, H. (2019). Predicting types of occupational accidents at construction sites in Korea using random forest model. Safety Science, 120, 226-236.

https://doi.org/10.1016/j.ssci.2019.06.034

Khakzad, N., Khan, F., \& Amyotte, P. (2012). Dynamic risk analysis using bow-tie approach. Reliability Engineering \& System Safety, 104, 36-44. https://doi.org/10.1016/j.ress.2012.04.003

Khakzad, N., Khan, F., \& Paltrinieri, N. (2014). On the application of near accident data to risk analysis of major accidents. Reliability Engineering \& System Safety, 126, 116-125. https://doi.org/10.1016/j.ress.2014.01.015

Lee, Y-C., Shariatfar, M., Rashidi, A., \& Lee, H-W. (2020). Evidence-driven sound detection for prenotification and identification of construction safety hazards and accidents. Automation in Construction, 113, 103-127. https://doi.org/10.1016/j.autcon.2020.103127

Li, G., Cao, H., \& Li, S. (1993). Structural dynamic reliability theory and application. Earthquake Press.

Li, S., Li, S., Zhang, Q., Xue, Y., Liu, B., Su, M., Wang, Z., \& Wang, S. (2010). Predicting geological hazards during tunnel construction. Journal of Rock Mechanics and Geotechnical Engineering, 2(3), 232-242. https://doi.org/10.3724/SP.J.1235.2010.00232

Mwakali, J. A. (2006). A review of causes and remedies of construction related accidents: The Uganda experience. In Proceedings from the International Conference on Advances in Engineering and Technology (pp. 285-299). https://doi.org/10.1016/B978-008045312-5/50032-7

Nnaji, C., \& Karakhan, A. A. (2020). Technologies for safety and health management in construction: Current use, implementation benefits and limitations, and adoption barriers. Journal of Building Engineering, 29, 101-212.

https://doi.org/10.1016/j.jobe.2020.101212

Rey, G., Clair, D., Fogli, M., \& Bernardin, F. (2011). Reliability analysis of roadway departure risk using stochastic processes. Mechanical Systems and Signal Processing, 25(4), 1377-1392. https://doi.org/10.1016/j.ymssp.2010.11.015

Ross, S. M. (1996). Stochastic processes (2nd ed.). John Wiley \& Sons. 
Sanni-Anibire, M. O., Mahmoud, A. S., Hassanain, M. A., \& Salami, B. A. (2010). A risk assessment approach for enhancing construction safety performance. Safety Science, 121, 15-29. https://doi.org/10.1016/j.ssci.2019.08.044

Shao, B., Hu, Z., Liu, Q., Chen, S., \& He, W. (2019). Fatal accident patterns of building construction activities in China. Safety Science, 111, 253-263. https://doi.org/10.1016/j.ssci.2018.07.019

Shohet, I. M., Luzi, M., \& Tarshish, M. (2018). Optimal allocation of resources in construction safety: Analytical-empirical model. Safety Science, 104, 231-238. https://doi.org/10.1016/j.ssci.2018.01.005

Stewart, M. G. (2001). Reliability-based assessment of ageing bridges using risk ranking and lifecycle cost decision analyses. Reliability Engineering and System Safety, 74(3), 263-273. https://doi.org/10.1016/S0951-8320(01)00079-5

Tang, C. T., Zhang, Z. H., \& He, Z. G. (2012). Research summary of bridge and tunnel construction safety risk evaluation method. Applied Mechanics and Materials, 209-211, 1402-1405.

https://doi.org/10.4028/www.scientific.net/AMM.209-211.1402

Williamson, E. B., \& Winget, D. G. (2005). Risk management and design of critical bridges for terrorist attacks. Journal of Bridge Engineering, 10(1), 96-106.

https://doi.org/10.1061/(ASCE)1084-0702(2005)10:1(96)

Winge, S., \& Albrechtsen, E. (2018). Accident types and barrier failures in the construction industry. Safety Science, 105, 158-166. https://doi.org/10.1016/j.ssci.2018.02.006

Winge, S., Albrechtsen, E., \& Mostue, B. A. (2019). Causal factors and connections in construction accidents. Safety Science, 112, 130-141. https://doi.org/10.1016/j.ssci.2018.10.015

Yang, H., Chew, D. A. S., Wu, W., Zhou, Z., \& Li, Q. (2012). Design and implementation of an identification system in construction site safety for proactive accident prevention. Accident Analysis \& Prevention, 48, 193-203. https://doi.org/10.1016/j.aap.2011.06.017

Yang, W.-J., \& Zhang, Z. (2011). Structural dynamic reliability study based on first-passage time probability analysis of continuous Markov processes. Engineering Mechanics, 28(7), 124-129.

Yu, H., Khan, F., \& Garaniya, V. (2015). Nonlinear Gaussian Belief Network based fault diagnosis for industrial processes. Journal of Process Control, 35, 178-200. https://doi.org/10.1016/j.jprocont.2015.09.004

Zhang, J., Zhang, W., Xu P., \& Chen N. (2019a). Applicability of accident analysis methods to Chinese construction accidents. Journal of Safety Research, 68, 187-196. https://doi.org/10.1016/j.jsr.2018.11.006

Zhang, W., Zhu, S, Zhang, X., \& Zhang, T. (2020a). Identification of critical causes of construction accidents in China using a model based on system thinking and case analysis. Safety Science, 121, 606-618. https://doi.org/10.1016/j.ssci.2019.04.038

Zhang, Z., Liu, X., Zhang, Y., Zhou, M., \& Chen, J. (2020b). Time interval of multiple crossings of the Wiener process and a fixed threshold in engineering. Mechanical Systems and Signal Processing, 135, 106389. https://doi.org/10.1016/j.ymssp.2019.106389

Zhang, Z., Zhou, M., \& Fang, M. (2019b). First-passage probability analysis of Wiener process using different methods and its applications in the evaluation of structural durability degradation. European Journal of Environmental and Civil Engineering. https://doi.org /10.1080/19648189.2019.1601134
Zhou, C., \& Ding, L. Y. (2017). Safety barrier warning system for underground construction sites using Internet-of-Things technologies. Automation in Construction, 83, 372-389. https://doi.org/10.1016/j.autcon.2017.07.005

Zhou, H., Zhao, Y., Shen, Q., Yang, L., \& Cai, H. (2020). Risk assessment and management via multi-source information fusion for undersea tunnel construction. Automation in Construction, 111, 103050. https://doi.org/10.1016/j.autcon.2019.103050

Zhu, Z., Park, M-W., Koch, C., Soltani, M., Hammad, A., \& Davari, K. (2016). Predicting movements of onsite workers and mobile equipment for enhancing construction site safety. Automation in Construction, 68, 95-101. https://doi.org/10.1016/j.autcon.2016.04.009 